\title{
C-arm Pose Estimation in Prostate Brachytherapy by Registration to Ultrasound
}

\author{
Pascal Fallavollita ${ }^{1}$, Clif Burdette ${ }^{2}$, Danny Song ${ }^{3}$, \\ Purang Abolmaesumi ${ }^{4}$, and Gabor Fichtinger ${ }^{1}$ \\ ${ }^{1}$ Queen's University, Canada \\ pascal@cs.queensu.ca \\ ${ }^{2}$ Acoustic MedSystems Inc., Illinois, USA \\ ${ }^{3}$ Johns Hopkins Hospital, Baltimore, USA \\ ${ }^{4}$ University of British Columbia, Canada
}

\begin{abstract}
In prostate brachytherapy, transrectal ultrasound (TRUS) is used to visualize the anatomy, while implanted seeds can be seen in C-arm fluoroscopy. Intra-operative dosimetry optimization requires reconstruction of the implanted seeds from multiple $\mathrm{C}$-arm fluoroscopy images, which in turn requires estimation of the $\mathrm{C}$-arm poses. We estimate the pose of the $\mathrm{C}$-arm by two-stage registration between the $2 \mathrm{D}$ fluoroscopy images to a 3D TRUS volume. As single-view 2D/3D registration tends to yield depth error, we first estimate the depth from multiple 2D fluoro images and input this to a single-view 2D/3D registration. A commercial phantom was implanted with seeds and imaged with TRUS and CT. Ground-truth registration was established between the two by radiographic fiducials. Synthetic ground-truth fluoro images were created from the CT volume and registered to the $3 \mathrm{D}$ TRUS. The average rotation and translation errors were $1.0^{\circ}\left(\mathrm{STD}=2.3^{\circ}\right)$ and $0.7 \mathrm{~mm}(\mathrm{STD}=1.9 \mathrm{~mm})$, respectively. In data from a human patient, the average rotation and lateral translation errors were $0.6^{\circ}\left(\mathrm{STD}=3.0^{\circ}\right)$ and $1.5 \mathrm{~mm}$ (STD=2.8 $\mathrm{mm}$ ), respectively, relative to the ground-truth established by a radiographic fiducial. Fully automated image-based C-arm pose estimation was demonstrated in prostate brachytherapy. Accuracy and robustness was excellent on phantom. Early result in human patient data appears clinically adequate.
\end{abstract}

\section{Introduction}

Prostate cancer is the second most common cancer in men, diagnosed in 192,280 new patients each year in North America [1]. Brachytherapy is a definitive treatment of early stage prostate cancer, chosen by over 50,000 men each year. The procedure entails permanent implantation of small radioactive isotope capsules (a.k.a. seeds) into the prostate to kill the cancer with radiation. Success hinges on precise placement of the implants to provide the needed dose distribution. Unfortunately, primarily due to tissue motion, organ deformation, and needle deflection, actual seed positions never turn out to be as planned. Intraoperative dose optimization during the procedure would allow for correcting deviations from the plan and thus tailor the dose to cancer without harming surrounding normal tissues. This requires localization of the prostate and implanted seeds; a much coveted function that is not available today [2]. Prostate 
brachytherapy is performed with transrectal ultrasound (TRUS) guidance that provides adequate real-time visualization of the prostate but not of the implanted seeds. Carm fluoroscopy is widely used for gross visual assessment of the implanted seeds (Figure 1) but it cannot show the prostate and other relevant structures. Fusion of these complementary modalities would enable dynamic dosimetry. A variety of implant reconstruction techniques have been investigated $[3,4,5]$ which share one common requirement: the relative poses of the fluoroscopy images must be known prior to reconstruction. The fluoroscopy pose is usually determined in one of three ways. (a) Electronic joint encoder [3], rarely available as most facilities do not upgrade their vintage $\mathrm{C}$-arms. (b) Optical tracker could localize the C-arm [4], which

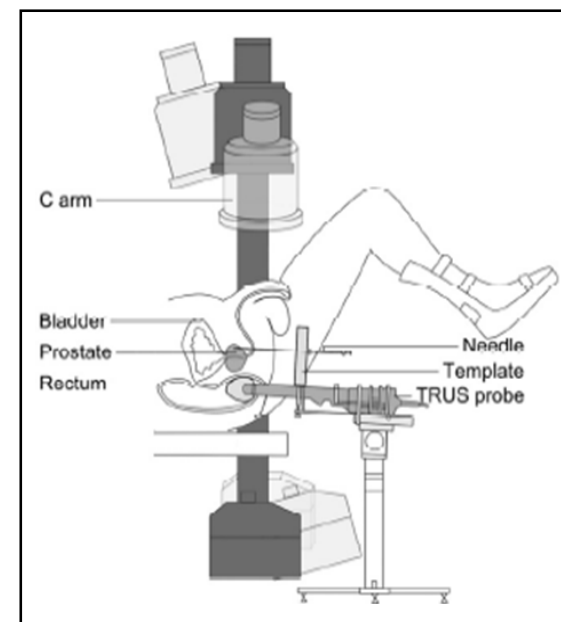

Fig. 1. Typical brachytherapy setup with TRUS probe and $\mathrm{C}$-arm with a narrow angular range. (Illustration by Xiao Xiao Ma). introduces prohibitive devices and logistical complexity in the otherwise streamlined clinical procedure. (c) Radiographic fiducials placed in the field of imaging, from which the pose of the fluoroscope can be discerned in relation to the fiducial structure [5]. Radiographic fiducials are independent of any C-arm brand or type. Unfortunately, fiducials must be segmented in fluoroscopy, a perennial issue for clinical practice. Also, the fiducials occupy priceless real estate in the image, forcing the prostate toward the edges where image distortion tends to be more severe, which in turn demands online distortion correction. Finally, mounting the fiducial to be visible in all $\mathrm{C}$-arm poses is a major procedural challenge and requires a large and thus very expensive image detector.

We propose a method that is radically different from the prior art. We estimate the relative pose of $\mathrm{C}$-arm images by the registration of the $2 \mathrm{D}$ fluoroscopy images to the 3D TRUS volume, and by doing so we estimate the poses of C-arm images in a coordinate system fixed to the prostate. As a byproduct, we receive an estimate of the registration between the $\mathrm{C}$-arm and ultrasound spaces, which is required for dynamic dosimetry. The objective is to recover the $\mathrm{C}$-arm poses with an accuracy that is sufficient for subsequent reconstruction of the implanted seeds like in [4] or [5].

Our contribution is the first report of $\mathrm{C}$-arm pose estimation by registration of $2 \mathrm{D}$ $\mathrm{C}$-arm images to 3D ultrasound. The apparent straightforwardness of our approach should not misrepresent the investment of creative effort needed to make it a workable clinical tool, despite the availability of underlying technical components. We devised an elegant and entirely novel solution for a longstanding clinical problem by adapting available techniques. Besides registering the $\mathrm{C}$-arm space directly to the prostate without surrogate markers, the most salient feature is that we avoid adding any instrumentation to the standard clinical setup. Our solution blends seamlessly with the current clinical install base and so it could be rapidly introduced to community care with minimal cost and make a positive impact in the very near future. 
Prior work in 2D/3D registration can be divided into two categories: feature-based and intensity-based methods. Feature-based methods [6] use distance between corresponding point pairs or surfaces as a measure to be optimized. Establishing point correspondences and minimizing the distance between them is alternated and repeated iteratively until convergence. Consequently, a segmentation of the data is required. Intensity-based methods compare the $2 \mathrm{D}$ image with a digitally reconstructed radiograph (DRR) created from the 3D volume. One can compare the imprints of anatomical structures obtained from either gradient information or voxel intensity [7-9]. Literature on registering a 3D ultrasound volume and 2D images has been scarce. Hummel et al. [10] used 2D ultrasound to 3D CT registration, where fiducial spheres served as markers for alignment. Leung et al. [11] reported rigid registration of 2D cardiac X-ray images with 3D echocardiography based on intensities, with crosscorrelation and sum of squared distances metrics. They also used a priori knowledge of the full pose to initialize the registration. They report rather large lateral translation and rotation errors of about $8 \mathrm{~mm}$ and $8^{\circ}$, respectively.

\section{Methodology}

\subsection{Central Intuition}

While seeds in an implanted prostate show up well in fluoroscopy, TRUS images of the prostate are saturated with artifacts emanating from seeds. In both modalities but especially in TRUS, artifacts often masquerade as seeds, an effect called false positive appearances. Seeds may also obscure one another in both modalities, an effect called hidden seeds. Although there is no exact matching between true positive appearances of seeds in TRUS and fluoroscopy, seeds carry enough common information for an intensity-based 2D/3D registration to "hone in" on the correct pose between the two. Due to false positives, exact segmentation of the seeds in TRUS is unattainable and it cannot be used for registration. Figure 2-left shows that even phantom images contain many false positives and there are more of them in human images.

\subsection{Single-View 2D/3D Registration}

We apply $2 \mathrm{D} / 3 \mathrm{D}$ registration considering the $3 \mathrm{D}$ TRUS as the moving volume and the 2D fluoro as the fixed image. As we only use one fluoro image at a time, we termed this single-view registration or shortly SVR.

Metric: We implemented the normalized cross correlation (NCC) metric that considers all pixel values in the images during registration. Fixed image pixels and their positions are mapped to the moving image. The correlation is normalized by the autocorrelations of both the fixed and moving images. Transform: After TRUS imaging, the probe is retracted from the rectum, so as not to block seeds during fluoroscopy. This causes the prostate to relax posteriorly, but usually without any apparent deformation. Good clinical practice requires minimal rectal pressure, to prevent deformation during TRUS imaging involving probe translation [2]. Prostate deformation is curtailed by minimizing rectal pressure. Since there is no delay between 3D TRUS and $2 \mathrm{D}$ fluoro acquisition, our assumption of 2D/3D rigid registration should suffice. 
We implemented a transformation of six parameters, three for Euler angles and three for translation. Initial Guess: In the operating room, we have an accurate and consistent initial guess for the registration. Standard patient positioning allows for aligning the main axes of the TRUS and the $\mathrm{C}$-arm. We also place the prostate around the center of the $\mathrm{C}$-arm that yields an estimate for the translation component of the transformation [5]. DRR Interpolator: A ray casting interpolator is used to project the moving TRUS volume onto the fixed 2D fluoro image. We cast

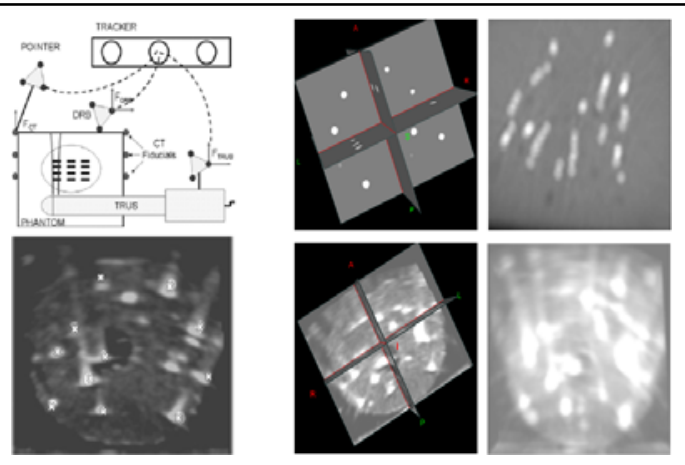

Fig. 2. (Left) Ground-truth phantom including coordinate systems and seeds in CT (x marks) overlaid on TRUS. The white blotches without corresponding seed are false positives. (Right) The synthetic fluoro DRR image obtained from the CT volume, as well as, an example image of the ray casted TRUS phantom.

rays from the $\mathrm{X}$-ray source of the

$\mathrm{C}$-arm through the moving TRUS volume to each pixel of the fixed fluoro image. As seeds in $\mathrm{C}$-arm show up as point-like structures we introduced a threshold during ray casting to ensure that only seed-like appearances from TRUS contribute to the DRR. Optimizer: The optimizer chosen to optimize the NCC similarity metric is the $(1+1)$ Evolutionary Strategy [13]. In this strategy, both the number of parents and the population size are set to one: $\mu=\lambda=1$. Mutation is accomplished by adding a vector of usually uncorrelated Gaussian random numbers, i.e. $\Sigma=\operatorname{diag}\left(\sigma^{2}\right)$ is a diagonal matrix. Step size adaptation can be performed according to Rechenberg's 1/5-rule: if less than $20 \%$ of the generations are successful then decrease the step size for the next generation; if more than $20 \%$ are successful, then increase the step size in order to accelerate convergence. As TRUS and fluoro are both spotted with positive appearances in lowsignal areas, the registration could be trapped in local minima. To counteract this problem, we restart the registration several times with slightly changing the initial pose and then take the median.

\subsection{Multi-view 2D/3D Registration}

As single-view 2D/3D registration is prone to depth error along the X-ray beam, we decided to bootstrap the registration in its weakest dimension, depth. In clinical practice, we can position the $\mathrm{C}$-arm so that the center of the prostate (judged by the extent of seeds cloud in the images) is near the C-arm's isocenter. With non-isocentric $\mathrm{C}$-arms, we can use coplanar rotation and set the axis of rotation in the prostate. With this setup, the prostate appears at a constant depth, especially at small (maximum $\pm 15^{\circ}$ ) rotations. We estimate this average depth from multiple fluoroscopy views. We pick pairs of fluoro images with wide angular separation and one-axis rotation between the two. We register them simultaneously to 3D TRUS in a much simplified manner, 
where we optimize only the depth and one rotation. The cost function implemented computes the sum of NCC between the fixed image and the DRR. Then we feed the approximate depth to the single-view $2 \mathrm{D} / 3 \mathrm{D}$ registration (Sec. 2.2.)

\subsection{Ground-Truth Phantom}

A commercial brachytherapy phantom (CIRS Inc., Virginia) was implanted with 48 non-radioactive Pd103 seeds according to a clinically realistic implant plan. Six CT fiducials were mounted on the opposite walls of the phantom, three on each side. This configuration of fiducials guarantees maximum target registration accuracy in the center where the prostate is located, Figure 2 (left). A dynamic reference body (DRB) optical marker (Traxtal, Versa Trax, model TT002-B) was affixed to the phantom, to define a coordinate frame that was used for constructing the $3 \mathrm{D}$ TRUS volume. The fiducials were localized with a calibrated pointer, relative to the DRB. The TRUS probe was tracked optically with Polaris (Northern Digital, Waterloo, Canada.), relative to the DRB. The CT fiducials were localized by segmentation and registered to their respective locations determined earlier by the tracked pointer. This yields ground-truth registration between the TRUS and CT.

\subsection{Ground-Truth 2D X-ray and TRUS Images}

In order to have perfectly accurate reliable ground-truth image poses, we decided to derive fluoroscopy images from high resolution CT volume image of the phantom. Further, the DRR$\mathrm{S}$ of the phantom computed are similar to $\mathrm{C}$-arm images, but with the immediate advantage that CT has higher geometrical accuracy with less experimental complexity. We acquired CT images of the phantom, with $0.3 \times 0.3 \mathrm{~mm}$ in-plane resolution and $0.6 \mathrm{~mm}$ slice thickness. As seeds are very prominent in $\mathrm{CT}$, we only clip a region of interest and use window-level scaling to

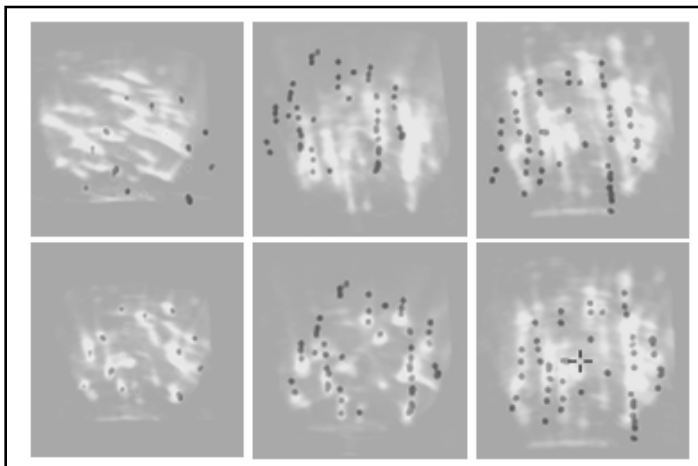

Fig. 3. Final overlay of TRUS DRR and CT data. (Top row) Initial perturbations before registration is initiated for angulations of $0^{\circ}, 15^{\circ}$, and $-15^{\circ}$ respectively. (Bottom row) Using the recovered depth from multi-view registration, a quasi perfect overlap is seen after the single-view registration. create the 8-bit CT. Window/level scaling is a method for mapping a range of intensity to a different scale. Generally, CT data has intensity values between -2000 to 4095. In our case, true seeds (intensities above 1700) were mapped to 8-bit grayscale. Then a suitable threshold of 100 was applied to suppress all remaining artifacts, thus yielding ground-truth seed locations in CT that was registered to TRUS earlier. We created DRR-s from the 8-bit CT, such as in Figure 2-right. The angular range of the 
$\mathrm{C}$-arm is constrained by the patient, table, or brachytherapy mount. In clinical practice, the maximum in-plane and out-of-plane rotation is about $\pm 15^{\circ}$. We created DRR-s at $15^{\circ}$ increments, using the geometry of a clinical C-arm from [5]. Again, the DRR-s played the role of the $2 \mathrm{D}$ fluoro images in subsequent phantom experiments. Using tracked freehand TRUS acquisition, we scanned the entire phantom volume systematically with translational motion, with continuous image capture. 2D pixel spacing was $0.14 \times 0.13 \mathrm{~mm}$. Interframe spacing was $0.5 \mathrm{~mm}$. An 8-bit 3D TRUS volume was compounded from the $2 \mathrm{D}$ images. A distinctive feature of our approach is that false positive seed appearances are allowed to remain in the TRUS data. As seeds create strong sonic impression in TRUS, as seen in Figure 2-right, it is not necessary to filter soft tissue signal from the images.

\section{Results and Discussion}

The clinical degree of accuracy for pose estimation and implant 3D reconstruction is presented in the paper by Jain et al. [12]; thus we require curtailing the rotation error to $\pm 4^{\circ}$ and lateral translations to about $\pm 2 \mathrm{~mm}$. In $2 \mathrm{D} / 3 \mathrm{D}$ registration, the cost metric usually has difficulties with properly "driving" the depth component of the pose. In $\mathrm{C}$-arm reconstruction, however, the exact same effect is working for our advantage, because the reconstruction metric is similarly insensitive to the depth component of the C-arm pose. Jain et al. [14] found that "reconstruction error is insensitive to miscalibration in origin and focal length errors of up to $50 \mathrm{~mm}$ ", inferring that huge depth errors are permissible if image poses shift together. What follows is that if the prostate is kept near the isocenter, projection and reconstruction are both insensitive to depth; a fact that we exploited in designing the multi-view registration scheme.

\subsection{Phantom Studies}

We used 20 random perturbations of maximum $\pm 5 \mathrm{~mm}$ translation and $\pm 5^{\circ}$ rotation about the ground-truth poses, and then we repeated the same with $\pm 10 \mathrm{~mm}$ and $\pm 10^{\circ}$. These perturbation values are justified since standard patient positioning constrains the rotation of the $\mathrm{C}$-arm and allows for quasi iso-centric positioning of the prostate. For each case we run a single-view (SVR) and multi-view (MVR) registration. The threshold in the DRR interpolator was set at 150, after trying several values. The results are summarized in Figure 3 and Table 1. All results are reported as absolute distances. With $\pm 5 \mathrm{~mm}$ and $\pm 5^{\circ}$ perturbations, SVR performed within clinical limits. All runs converged and are reported. Average rotation and lateral translation (Tx/Ty) errors were $0.8^{\circ}\left(\mathrm{STD}=2.3^{\circ}\right)$ and $0.5 \mathrm{~mm}(\mathrm{STD}=1.6 \mathrm{~mm})$, respectively. The depth error $(\mathrm{Tz})$ was $3.5 \mathrm{~mm}(\mathrm{STD}=3.5 \mathrm{~mm})$. Then MVR reduced the depth error to $1.2 \mathrm{~mm}$ $(\mathrm{STD}=2.8 \mathrm{~mm})$. Note that lateral translation and rotation errors remained about the same, as MVR affects only the depth (Section 2.3.) With $\pm 10 \mathrm{~mm}$ and $\pm 10^{\circ}$ perturbation, pose recovery was less accurate. Still, all runs converged and are reported. SVR achieved average rotation and lateral translation errors of $3.8^{\circ}\left(\mathrm{STD}=3.4^{\circ}\right)$ and 2.3 $\mathrm{mm}(\mathrm{STD}=2.1 \mathrm{~mm})$, respectively. Then MVR reduced the depth error to $2.9 \mathrm{~mm}$ $(\mathrm{STD}=2.1 \mathrm{~mm})$. 
Table 1. Ground-truth phantom results. Average translation and rotation error with STD, for single-view and multi-view registration.

\begin{tabular}{|c|c|c|c|c|c|c|}
\hline \multirow[b]{3}{*}{ SVR } & \multicolumn{3}{|c|}{ $\pm 5 \mathrm{~mm}$ and $\pm 5^{\circ}$ perturbation } & \multicolumn{3}{|c|}{ $\pm 10 \mathrm{~mm}$ and $\pm 10^{\circ}$ perturbation } \\
\hline & Tx/Ty & $\mathbf{T z}$ & Rotation & Tx/Ty & $\mathbf{T z}$ & Rotation \\
\hline & $0.5 \pm 1.6$ & $3.5 \pm 3.5$ & $0.8 \pm 2.3$ & $2.3 \pm 2.1$ & $5.7 \pm 4.7$ & $3.8 \pm 3.4$ \\
\hline MVR & $0.4 \pm 1.5$ & $1.2 \pm 2.8$ & $1.0 \pm 2.3$ & $2.2 \pm 2.4$ & $2.9 \pm 2.1$ & $3.9 \pm 3.4$ \\
\hline
\end{tabular}

We implemented the single-view and multi-view registration technique using the Insight toolkit (ITK). We used an Intel Core2, 2.4 GHz dual-core computer. The average speed of the SVR registration was 60 seconds which is feasible in clinic.

\subsection{Clinical Results}

Clinical patient data was collected under ethics board approval. Here we report results on the first patient dataset in the trial. Nine C-arm fluoroscopy images were acquired and their relative poses recovered with a precision-machined radiographic fiducial [5], serving as ground-truth. For the 2D/3D registration, the fluoro images were dewarped and a $256 \times 256$ pixel ROI was cut around the prostate's center. The registration parameters were retuned: since the rotation has a stronger initial guess in the actual clinical setup we assigned higher optimization weights to rotation than to translation. We used 20 random perturbations of maximum $\pm 5 \mathrm{~mm}$ translation and $\pm 5^{\circ}$ rotation about the true poses, and then we repeated the same with $\pm 10 \mathrm{~mm}$ and $\pm 10^{\circ}$. We only ran the single-view registration (SVR). MVR could not be tested, because the fiducial prevented us from setting the prostate in the isocenter. At $\pm 5 \mathrm{~mm}$ and $\pm 5^{\circ}$ perturbations, human patient data, the average rotation and lateral translation errors were $0.6^{\circ}$ $\left(\mathrm{STD}=3.0^{\circ}\right)$ and $1.5 \mathrm{~mm}(\mathrm{STD}=2.8 \mathrm{~mm})$, respectively, relative to the ground-truth established by a precise radiographic fiducial. The average depth recovered was 3.8 $\mathrm{mm}(\mathrm{STD}=4.2 \mathrm{~mm})$. After doubling the maximum perturbation, the average rotation and lateral translation errors were $1.7^{\circ}\left(\mathrm{STD}=5.7^{\circ}\right)$ and $1.4 \mathrm{~mm}(\mathrm{STD}=4.0 \mathrm{~mm})$, and average depth of $5.1 \mathrm{~mm}(\mathrm{STD}=6.7 \mathrm{~mm})$. Although average errors grew, they still remained below the clinically acceptable limits.

Visual observation is not sufficient for more precise evaluation, due to the concurrent effects of true seeds, false positives and hidden seeds. When these are all compounded in ray casting, the best matching is not perceivable to the human eye. Nonetheless, true seeds carry sufficient information for the intensity-based metric to lock on the pose. This phenomenon underscores why explicit segmentation of the seeds in transrectal ultrasound cannot be used for registration or for the evaluation thereof. Our experience with early clinical data clearly and forcefully underlines the inherent difficulty of reliable validation based on explicit segmentation of seeds in TRUS. For many seeds, the expert clinician could not tell apart true seeds from noise in TRUS. After two weeks, the clinician repeated the task of seed identification in the same patient data and nearly half of all seed locations were picked differently, suggesting unreliable consistency in visual seed localization. A possible workaround might be applying multiple segmenters, but that is likely to fail as well. Earlier, Orio et al. reported the same difficulty [15], as they were able to visually identify $20-25 \%$ of all the implanted seeds in TRUS. We established registration ground-truth as suggested by Jain et al. in [5], by pre-registration of a 
radiographic fiducial and the TRUS coordinate space. This approach, however, is not generally robust and, as Jain et al. mentioned, it may require compensation for biases emanating from multiple sources.

In summary, we presented the first application of $2 \mathrm{D} / 3 \mathrm{D}$ registration in prostate brachytherapy for estimating the $\mathrm{C}$-arm pose. Overall accuracy and robustness were excellent on phantom data and adequate on human data. The ultimate test, still, is whether this pose estimation will prove to be sufficient in brachytherapy implant reconstruction such as [4] or [5] - an issue of great clinical interest.

\section{References}

1. Jemal, A., Siegel, R., Ward, E., et al.: Cancer Statistics. Cancer J. Clin. 59(4), 225-249 (2009)

2. Nag, S., Ciezki, J.P., Cormack, R., et al.: Intraoperative planning and evaluation of permanent prostate brachytherapy: report of the American Brachytherapy Society. Int. J. Radiat. Oncol. Biol. Phys. 51(5), 1422-1430 (2001)

3. Westendorp, H., Hoekstra, C.J., van't Riet, A., et al.: Intraoperative adaptive brachytherapy of iodine- 125 prostate implants guided by C-arm cone-beam computed tomographybased dosimetry. Brachytherapy 6(4), 231-237 (2007)

4. Su, Y., Davis, B.J., Furutani, K.M., et al.: Seed localization and TRUS- fluoroscopy fusion for intraoperative prostate brachytherapy dosimetry. Computer Aided Surgery 12(1), 25 34 (2007)

5. Jain, A.K., Deguet, A., Iordachita, I., et al.: Intra-operative Guidance in Prostate Brachytherapy Using an Average Carm. In: Ayache, N., Ourselin, S., Maeder, A. (eds.) MICCAI 2007, Part II. LNCS, vol. 4792, pp. 9-16. Springer, Heidelberg (2007)

6. Yamazaki, T., Watanabe, T., Nakajima, Y., et al.: Improvement of depth position in $2 d / 3 d$ registration of knee implants using single-plane fluoroscopy. IEEE Transactions on Medical Imaging 23(5), 602-612 (2004)

7. Livyatan, H., Yaniv, Z., Joskowicz, L.: Gradient-based 2-D/3-D rigid registration of fluoroscopic X-ray to CT. IEEE Trans. Med. Imaging 22, 1395-1406 (2003)

8. Mahfouz, M., Hoff, W., Komistek, R., Dennis, D.: A robust method for registration of three-dimensional knee implant models to two-dimensional fluoroscopy images. IEEE Transactions on Medical Imaging 22, 1561-1574 (2003)

9. Lau, K., Chung, A.: A global optimization strategy for 3d-2d registration of vascular images. In: Proceedings of 17th British Machine Vision Conference, pp. 489-498 (2006)

10. Hummel, J., Figl, M., Bax, M., Bergmann, H., Birkfellner, W.: 2D/3D registration of endoscopic ultrasound to CT volume data. Physics in Medicine and Biology 53(16), 4303-4316 (2008)

11. Leung, K.Y.: Registration of $2 \mathrm{D}$ cardiac images to real-time 3D ultrasound volumes for 3D stress echocardiography. In: SPIE Medical Imaging, vol. 6144, pp. 405-416 (2006)

12. Jain, A., Fichtinger, G.: C-arm Tracking and Reconstruction without an External Tracker. In: Larsen, R., Nielsen, M., Sporring, J. (eds.) MICCAI 2006. LNCS, vol. 4190, pp. 494 502. Springer, Heidelberg (2006)

13. Arnold, D.V., Brauer, D.: Local performance of the $(1+1)$-ES in a noisy environment. IEEE Transactions on evolutionary computation 6(1), 30-41 (2002)

14. Jain, A., Kon, R., Zhou, Y., Fichtinger, G.: C-arm calibration - is it really necessary? In: Duncan, J.S., Gerig, G. (eds.) MICCAI 2005. LNCS, vol. 3749, pp. 639-646. Springer, Heidelberg (2005)

15. Orio, P.F., Tutar, I.B., Narayanan, S., et al.: Intraoperative ultrasound-fluoroscopy fusion can enhance prostate brachytherapy quality. Int. J. Radiat. Oncol. Biol. Phys. 69(1), 302-307 (2007) 